\title{
Research Progress on Treatment of Ulcerative Colitis with Baitouweng Decoction
}

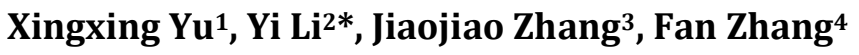 \\ ${ }^{1}$ The First Clinical Medical College of Shaanxi University of Traditional Chinese Medicine, Xianyang 712000, Shaanxi \\ Province, China \\ ${ }^{2}$ Affiliated Hospital of Shaanxi University of Traditional Chinese Medicine, Xianyang 712000, Shaanxi Province, China \\ ${ }^{3}$ The First Clinical Medical College of Shaanxi University of Traditional Chinese Medicine, Xianyang 712000, Shaanxi \\ Province, China \\ ${ }^{4}$ The First Clinical Medical College of Shaanxi University of Traditional Chinese Medicine, Xianyang 712000, Shaanxi \\ Province, China \\ *Corresponding author: Yi Li, $184697086 @ q q . c o m$

\begin{abstract}
The research literature, including the overview, clinical research and experimental research, about the treatment of ulcerative colitis (UC) with Pulsatilla Decoction was reviewed in this paper in order to provide reference for its development and application.
\end{abstract}

Keywords: Pulsatilla Decoction; Ulcerative colitis; Clinical studies; Experimental studies

Publication date: July 2021; Online publication: July 30, 2021

\section{Introduction}

Ulcerative colitis (UC) is an idiopathic autoimmune disease that easily affects the colon and rectum. Its etiology and pathogenesis are still unclear ${ }^{[1]}$. In recent years, biological agents and microecologics have been gradually applied to clinical treatment, but it is difficult to avoid recurrence and obvious adverse reactions after drug discontinuation in Western medicine ${ }^{[2]}$. Traditional Chinese medicine (TCM) has enriched source for the treatment of UC that has the significant advantages of low recurrence rate and less adverse reactions. Baitouweng Decoction is a famous prescription in Shanghanlun and has a significant effect in the clinical treatment of UC. In this article, we summarize the advantages of clinical application of medicine in UC so as to better promote the application of the classic prescriptions.

\section{Overview of Baitouweng Decoction}

For a long time, Baitouweng Decoction has been a famous prescription for the treatment of diarrhea, which is derived from Treatise on Febrile Diseases. In ancient times, it was often used to treat diarrhea and other diseases. In his book, Zhongjing Zhang recorded that "those who warm up and benefit the spirit, the Pulsatilla soup is the master." This prescription is made up of four Chinese medicinal materials, i.e. Pulsatillae Radix, Coptidis Rhizoma, Cortex phellodendri, and Cortex Fraxini, and has the effects of clearing away heat and toxic materials, relieving diarrhea and pain, and improving blood circulation. 


\section{Clinical study of Baitouweng Decoction in the treatment of UC}

\subsection{Clinical efficacy of Pulsatilla Decoction in the treatment of UC}

Yang ${ }^{[3]}$ conducted a study in which the control subjects orally took modified Pulsatilla Decoction and experimental subjects orally took Western medicine for treatment, and found that compared with the latter, the former could reduce the TCM syndrome scores, promote the early repair of ulcer surface, improve the curative effect and prognosis. Ma et al. found a better effect of mesalazine combined with Pulsatilla Decoction ${ }^{[4]}$. Bi found that the combination of Baitouweng Decoction and acupuncture treatment could reduce the inflammatory reaction and promote the immune function recovery ${ }^{[5]}$. According to Tian et al., comparison of the patients who took mesalazine orally using Pulsatilla Decoction and those who took Chinese medicine enema as control revealed that the effect of the Chinese medicine group was better after continuous treatment for 8 weeks ${ }^{[6]}$. The research conducted by the above-mentioned experts has shown that irrespective of oral administration of Baitouweng Decoction alone or in combination with western medicine, acupuncture and moxibustion treatment as well as the commonly used enema therapy of Baitouweng Decoction in clinic has achieved significant curative effects. In addition, the adverse reactions caused by the above treatment methods are significantly reduced, and the recurrence rate is significantly reduced, indicating that this prescription is worthy of clinical promotion and application.

\subsection{Expert experience of modified Pulsatilla Decoction in the treatment of UC}

Based on the "theory of dampness and turbidity," Chinese medicine practitioner Diangui Li formulated the combined prescription that eliminates turbidity and detoxicates for the treatment of UC, which had good efficacy ${ }^{[7]}$ : Professor Jialin Wang attaches great importance toregulating the balance of yin and yang of human body, so he made his own modified Pulsatilla Decoction to treat UC with good effect ${ }^{[8]}$. Professor Jingen Lu believed that mild and moderate UC in the active stage of TCM clinical classification was mostly classified into large intestine damp-heat and spleen deficiency dampness syndrome, so the use of Pulsatilla Decoction combined with Caulis Sargentodoxae Powder to treat UC was found to achieve good outcomes

[9]. Professor Jingri Xie found that the use of Rhizoma Coptidis Jiedu Decoction combined with Radix Pulsatillae Decoction to protect spleen and stomach in the treatment process has very good effect ${ }^{[10]}$.

\section{Experimental study on the treatment of UC with Baitouweng Decoction}

\subsection{Regulation of cytokines}

Studies have shown that Radix Pulsatillae has medicinal effects on UC by maintaining the balance of cytokines. Li et al. found that Baitouweng Decoction could regulate the pathological morphology of the colon, down-regulate the amount of interleukin (IL)-17A and up-regulate the amount of IL-10 ${ }^{[11]}$. Ge et al. found that compared with enema with other drugs, the enema with modified Baitouweng Decoction could reduce the levels of pro-inflammatory factors IL- 8 and transforming growth factor-beta1 (TGF- $\beta 1$ ) and increase the anti-inflammatory factor IL-13 level, thus promoting the healing of ulcers ${ }^{[12]}$. Liu et al. found that Baitouweng Decoction could reduce the activity of nuclear factor kappa B $(\mathrm{NF}-\kappa \mathrm{B})$ p65 and promote the balance of cytokines to exert the therapeutic effect on UC ${ }^{[13]}$.

\subsection{Regulation of signaling pathways}

$\mathrm{Li}$ et al. found that Baitouweng Decoction might regulate the pathways, including Toll-Like receptors (TLRs), NF- $\kappa \mathrm{B}$, NOD-like receptors (NLRs), 5-hydroxytryptamine (5-HT), and arachidonic acid (AA), through multiple targets, thereby reducing inflammation damage ${ }^{[14]}$. Song et al. found that, based on experimental research, Baitouweng Decoction might inhibit and protect the intestinal mucosa damage through two proteins of epidermal growth factor receptor (EGFR) and fibronectin (FN), as well as two signaling pathways of mitogen-activated protein kinase (MAPK) and phosphoinositide 3-kinase (PI3K), 
and thus treat $\mathrm{UC}{ }^{[15]}$. $\mathrm{Hu}$ et al. demonstrated that the NF- $\mathrm{BB} / \mathrm{COX}-2$ signaling pathway played an important role in the formation of UC, and aesculin in Pulsatilla Decoction could inhibit the inflammatory process by inhibiting the activity of this pathway ${ }^{[16]}$. Miu et al. have found through experiments that there are many signaling pathways in the treatment of UC with Baitouweng Decoction, which mostly work by regulating the proliferation and regulation of cells ${ }^{[17]}$. Ou et al. found that Baitouweng Decoction was closely related to two major signaling pathways during the treatment process, namely Leucocyte Transendothelial Migration and P13K-AKT; the former helps to reduce inflammation, while the latter helps to regulate biological processes ${ }^{[18]}$. Li et al. found that modified Baitouweng Decoction could inhibit the over-activated NF- $\kappa \mathrm{B}$ in the UC rat model ${ }^{[19]}$.

\subsection{Reduction of inflammatory mediators}

Baitouweng Decoction reduced the content of inflammatory mediators in UC model rats, thereby reducing the inflammatory damage and inhibiting intestinal inflammation. Yang et al. found a significant effect of modified Baitouweng Decoction on inflammatory response in rats with UC in their research ${ }^{[20]}$. On the one hand, it can reduce the serum intercellular adhesion molecule 1 (ICAM-1) level, and on the other hand, it can reduce the serum p-selectin level, thus reducing the inflammatory response and promoting mucosal repair.

\subsection{Regulation of immune function}

Baitouweng Decoction can normalize the immune function of UC model rats by regulating immune function, thus playing a role in the treatment of UC. Liu et al. showed that Baitouweng Decoction was likely to play a role by regulating the balance of cytokine network ${ }^{[21]}$. The higher the degree of inflammation is, the higher the expression level of adhesion factors CD54 and CD44 will be. Baitouweng Decoction recovers immune function through the above-mentioned pathways, and then the expanded inflammatory response is inhibited in order to treat UC. Wang et al. found that the expression of each component of NLRP3 inflammasomes played a central role in the treatment process, and these components played a role by regulating the expression of downstream proteins, such as IL-18 and IL-1 ${ }^{[22]}$.

\subsection{Repair of mucosal barrier}

At present, there are many factors governing the occurrence and development of UC, and the dysfunction of mucosal barrier is closely related to its pathogenesis, which has been proven by experiment ${ }^{[23]}$.

\section{Conclusion}

Oral administration of Pulsatilla Decoction, combined with western medicine, enema and acupuncture are common methods for treating UC. Modern clinical and experimental results show that Pulsatilla Decoction has definite curative effect, less adverse reactions and low recurrence rate. However, more research is needed. Therefore, we should further study the pharmacodynamics and mechanism of Pulsatilla Decoction in the treatment of UC in order to provide basis for clinical treatment.

\section{Disclosure statement}

The author declares no conflict of interest.

\section{References}

[1] Shen H, Tang Z, Tang X, et al., 2019, Digestive System Common Disease Ulcerative Colitis TCM Diagnosis and Treatment Guidelines (Grassroots Doctors Version). Chinese Journal of Traditional 
Chinese Medicine, 34(9):4155-4160.

[2] Xiong B, Fang S, 2020, Research Progress on Clinical Treatment of Ulcerative Colitis. Chinese Medical Journal, 22(3): 170-173.

[3] Yang J, 2020, Baitouweng Decoction in the Treatment of Ulcerative Colitis Large Intestine DampHeat Type Efficacy Analysis. Journal of Practical Traditional Chinese Medicine, 4: 412-413.

[4] Ma Y, Hou F, Li H, 2020, Baitouweng Decoction Combined with Western Medicine in the Treatment of Large Intestine Damp-Heat Ulcerative Colitis. Journal of Traditional Chinese Medicine, Liaoning, 1: 114-116.

[5] Bi W, 2020, Baitouweng Decoction Combined with Acupuncture and Moxibustion Treatment of Ulcerative Colitis on the Patient's Immune Function and IL-1 $\beta$, IL-17 Effects. Electronic Journal of Modern Medicine and Health Research, 10: 96-98.

[6] Tian LJ, 2020, Oral Administration of Baitouweng Decoction Combined with Chinese Medicine Enema in the Treatment of Ulcerative Colitis. Modern Chinese Medicine Application, 14(8): 210 211.

[7] Bai H, Li N, Yang Z, et al., 2019, Li Diangui’s Experience in Treating Ulcerative Colitis Based on Turbidity Toxin Theory. Shanghai Journal of Traditional Chinese Medicine, 53(4):2-4+1.

[8] Xu SY, Sun Y, Zhou L, et al., 2018, Professor Wang Jialin's Experience in Treating Ulcerative Colitis with Baitouweng Decoction. Modern Chinese Medicine Clinical, 25(5): 35-37.

[9] Chen B, Chang Z, Lu J, 2020, Professor Lu Jingen Treatment of Mild and Moderate Ulcerative Colitis Academic Thinking. Western Medicine, 33(2): 47-49.

[10] Fang Y, Zhang B, 2015, Xie Jingri's Experience in the Treatment of Ulcerative Colitis. Henan Traditional Chinese Medicine, 35(5): 954-955.

[11] Li P, Li D, Li Y, et al., 2020, Baitouweng Decoction on Ulcerative Colitis Model Rats IL-17A and IL-10 Effects. Chinese Medicine Guide, 2: 1-4.

[12] Ge M, Wu Y, Hsuan C, 2019, Modified Baitouweng Decoction Enema in the Treatment of Active Damp-Heat Ulcerative Colitis in Rats. Laboratory Medicine and Clinical, 7: 934-938.

[13] Liu W, 2016, Correlation Between O-GlcNAc and IBD and Intervention Mechanism of Pulsatilla Decoction on IBD. Yangzhou University.

[14] Li P, Luo Y, Gu S, et al., 2020, To Explore the Mechanism of Pulsatilla Decoction in the Treatment of Ulcerative Colitis Based on Network Pharmacology. Chinese Journal of Integrated Traditional and Western Medicine on Digestion, 7: 527-533.

[15] Song H, Chen X, Zeng M, et al., 2020, Molecular Network Regulation of Baitouweng Decoction in the Treatment of Ulcerative Colitis. Chinese Journal of Hospital Pharmacy, 40(10): 1098-1105.

[16] Hu Y, Yang S, Wang A, et al., 2020, To Explore the Mechanism of Baitouweng Decoction in the Treatment of Ulcerative Colitis. Pharmacology and Clinical Research of Traditional Chinese Medicine, 1: 55-60.

[17] Miu Z-W, Xu Y, Ning L-Q, et al., 2020, Network Pharmacology Analysis and Preliminary Verification of Molecular Mechanism of Baitouweng Decoction in the Treatment of Ulcerative Colitis. Chinese Journal of Traditional Chinese Medicine, 45(8): 1808-1815.

[18] Ou H-Y, Ye X, Ji Y-C, et al., 2019, Study on the Mechanism of Baitouweng Decoction in the 
Treatment of Ulcerative Colitis Based on Network Pharmacology. Chinese Journal of Traditional Chinese Medicine, 37(10): 2438-2443+2572-2573.

[19] Li Y, He J, 2013, Effect of Modified Baitouweng Decoction on NF- $\kappa$ B Expression in Rats with Ulcerative Colitis. Guangming TCM, 28(10): 2056-2059.

[20] Yang J, 2016, Experimental Study on the Treatment of Ulcerative Colitis Model Rats with DampHeat Retention in the Intestine with Modified Baitouweng Decoction. Liaoning University of Traditional Chinese Medicine.

[21] Liu J, Qiu M, Tao C, et al., 2010, Effects of Modified Baitouweng Decoction on Colonic Cytokines and Adhesion Factors in Rats with Ulcerative Colitis. Chinese Journal of Integrated Traditional and Western Medicine on Digestion, 18(1): 30-33.

[22] Wang Z, Sun P, Li T, et al., 2012, Baitouweng Decoction Activates NLRP3 Inflammasomes in the Treatment of Inflammatory Bowel Disease. Chinese Medicinal Materials, 35(8): 1280-1286.

[23] Xabier RJ, Podolsky DK, 2007, Unraveling the Pathogenesis of Inflammatory Bowel Disease. Nature, 448: 427-434. 